\title{
Effect of Collaboration on Localizability in Range-Based Localization Systems
}

\author{
Javier Schloemann, Harpreet S. Dhillon, and R. Michael Buehrer
}

\begin{abstract}
In this paper, we examine, via analysis, the improvement in device localizability through collaboration. Depending on the sensitivity of the receivers in the devices, it is not unusual for an unlocalized device to lack a sufficient number of detectable positioning signals from localized devices to determine its location without ambiguity (i.e., to be uniquely localizable). This occurrence is well-known to be a limiting factor in localization performance, especially in communications systems. In cellular positioning, for example, cellular network designers call this the hearability problem. In this work, we employ tools from stochastic geometry to derive accurate approximations for the probabilities of unique localizability in the noncollaborative and collaborative cases. We consider range-based positioning scenarios with and without shadowing. The results are very promising and motivate further research into enhancing cellular positioning with smallscale collaboration (e.g., using D2D in LTE).
\end{abstract}

Index Terms - Collaborative localization, unique localizability, hearability, stochastic geometry, point process theory.

\section{INTRODUCTION}

Determining the locations of devices in mobile ad-hoc networks (MANETs), wireless sensor networks (WSNs), and cellular networks has many important applications such as location-aided routing [1], geodesic packet forwarding [2], network self-organization [3], tying sensor observations to locations [4], location-based services, and providing public service answering points with potentially life-saving location information during emergency calls [5]. Using prevalent global navigation satellite systems (GNSS), e.g., GPS, is often the preferred method of location estimation; however, such systems are not always available or reliable. For example, wireless devices are often used indoors where satellite signals may be too weak to provide reliable location estimates. Further, with the advent of new indoor location requirements imposed by the Federal Communications Commission (FCC) [6], the indoor case is becoming increasingly important for cellular network operators.

Classically, the localization procedure is performed separately at the mobile devices (MDs), each communicating only with a set of already-localized reference devices, which we will call base stations (BSs). The first objective in any such location system is to make sure that the MDs can receive positioning signals from a sufficient number of BSs in order to calculate position fixes. This is far from guaranteed; in fact, cellular network designers call this the hearability problem [7]. In recent years, collaboration between MDs has received more and more attention as a means to improve positioning performance,

The authors are with Wireless@ Virginia Tech, Blacksburg, VA, USA. Email: \{javier, hdhillon, buehrer\}@vt.edu. both for MANETs and WSNs [8], [9] as well as cellular networks [10]. The primary benefits provided by collaboration are (i) an increased ability to calculate position fixes [11] and (ii) more accurate position fixes [12]. This paper studies the former benefit by asking: how does enhancing a classical rangebased localization procedure with a single collaborative link impact the availability of position fixes?

There is a rich body of literature concerning the study of MANETs and WSNs, both for connectivity as well as positioning. For these networks, it is standard to model device locations according to a homogeneous Poisson point process (PPP) [13], since deployments are not rigidly planned. From the localization perspective, a substantial portion of previous work is focused on collaborative localization, whereby MDs gather position-related observations not just from BSs, but also from each other. Analysis is known to be hard and the majority of papers eventually resort to simulation (e.g., [14], [15]). The analysis in [12] shows that enhancing a classical localization procedure with even just one collaborative link will strictly reduce the Cramér-Rao lower bound (CRLB) of the positioning error. Due to its formulation, however, a CRLB analysis cannot take into account whether or not a device is unable to uniquely locate itself due to ambiguities (such as the flip ambiguity [16]). Thus, as a complement to [12] which studied how collaboration impacts positioning accuracy, this work studies how collaboration impacts positioning availability.

The cellular network literature is also very rich with connectivity analyses (in the form of coverage probabilities) and with the proliferation of smart phones enabling locationbased services as well as increased pressure due to federal regulations (e.g., the FCC E911 mandate [17]), positioning of cellular devices has recently garnered increasing interest. Due in large part to the fact that cellular networks are usually modeled using widely-accepted grid-based models, which do not lend themselves to tractable analyses, the literature typically relies on simulation. An example is [10], which used simulation results to show that collaboration provides an increase in the availability of positioning fixes in LTE networks using OTDOA. A recent trend, however, is to model cellular BS deployments according to a homogeneous PPP (e.g., [18], [19]), which reasonably model actual BS deployments while greatly increasing analytical tractability. This approach was taken in [20], which studied the hearability of far away BSs for the purposes of cellular positioning. Among other things, the results in [20] can be used to determine the probability that a device will be unable to locate itself without ambiguity using a classical localization technique. The present work expands 
on this and studies how often a collaborative link can help a device locate itself without ambiguity.

Thus, the objective of this paper is to explicitly consider the following question: how does the presence of a collaborative link affect a mobile device's ability to locate itself without ambiguity? BSs are modeled according to a homogeneous PPP. The MDs are modeled in the same way, using a second (independent) PPP, which agrees with the uniformly-random modeling of MDs in MANETs, WSNs, 3GPP simulations. Our model considers network self-interference, something which is often omitted from MANET and WSN studies Using a baseline pathloss-only propagation model (common for MANETs and WSNs), analytical expressions for the probabilities of localizability in range-based positioning are presented. The analysis is then extended to consider the impact of shadowing, commonly included in cellular propagation models.

\section{SySTEM MODEL}

The locations of the BSs and MDs are modeled using two independent homogeneous PPPs $\Phi, \Psi \in \mathbb{R}^{2}$ with densities $\lambda, \nu$ [13], respectively. If the interference is treated as noise at the receiver, the most appropriate metric that captures link quality is the signal-to-interference-plus-noise ratio (SINR). For the link from some BS $x \in \Phi$ to some MD $z \in \Psi$, the SINR can be expressed as:

$$
\operatorname{SINR}_{x \rightarrow z}=\frac{P \mathcal{F}_{x \rightarrow z}\|x-z\|^{-\alpha}}{\sum_{\substack{y \in \Phi \\ y \neq x}} P \mathcal{F}_{y \rightarrow z}\|y-z\|^{-\alpha}+\sigma^{2}},
$$

where $P$ is the transmit power, $\mathcal{F}_{g \rightarrow h}$ denotes the slow fading coefficient from BS $g$ to MD $h, \alpha>2$ is the pathloss exponent, and $\sigma^{2}$ is the noise variance at the receiver.

To improve the hearability of far away BSs, positioning systems work at lower SINRs than communications systems, thereby necessitating the need for processing gain, which will depend upon the signal integration time. As a side effect, the processing averages out the effect of small-scale fading. Thus, the SINR does not contain a fast fading term, which is consistent with common models for evaluating cellular positioning performance [21].

It is well-known that including an increasing number of BSs in the localization procedure results in a general improvement in positioning accuracy. Thus, we assume that a device will take advantage of as many BSs as it can successfully detect (or hear). Specifically, a MD $z$ includes a BS $x$ in its localization procedure when

$$
\operatorname{SINR}_{x \rightarrow z} \geq \beta,
$$

where $\beta$ is the SINR threshold (prior to any processing gain) above which the signals from the BSs must arrive in order for them to be detected (i.e., this is the hearability condition). In the presence of shadowing, the set of included BSs at $z$ will not necessarily correspond to those which are geographically closest to $z$.

In this analysis, we consider the impact of extending a classical range-based location estimation procedure with a single collaborative ranging observation. Formally, we define the classical localization procedure as one where an unlocalized device communicates only with BSs, gathers position-related observations and solves the resulting single-location estimation problem to determine its location. For MANETs and WSNs, this type of setup corresponds to that employed in [22]. For cellular networks, this could correspond to downlink range-based positioning methods. When a classical localization procedure is extended by a collaborative link, the estimation problem then consists of two unknown device locations which are estimated simultaneously. While more complex, the addition of a collaborative link may allow a node which is otherwise not able to locate itself using the classical procedure to be able to uniquely determine its location. Understanding exactly how often collaboration helps is the purpose of this study.

The following notation is used throughout this paper: $\mathcal{A} \backslash \mathcal{B}$ represents the set (or area) $\mathcal{A}$ excluding $\mathcal{B} ;\|\mathcal{A}\|,\|\mathbf{z}\|$ is the Lebesgue measure of region $\mathcal{A}$ or the $\ell_{2}$-norm of vector $\mathbf{z} ;|\mathcal{S}|$ represents the cardinality of set $\mathcal{S}$; and $\mathbb{1}(\cdot)$ is the indicator function, i.e., it returns $1 / 0$ when its argument is true/false.

\section{UNIQUE LOCALIZABILITY}

The first objective in any localization system is to make sure that the devices to be located can be uniquely localized [23].

Definition 1 (Unique device localizability). An MD is uniquely localizable, if an estimate of the device's location can be found without ambiguity. In the noiseless case, this means that there can only be one solution to the set of non-linear equations that relate the observations to the unknown position. In the noisy case, this means that there is a single global minimum to the cost function.

For classical positioning techniques based on observations between the MD and the BSs, it is widely-accepted that the unique localizability condition simplifies to whether or not a mobile device is able to hear a sufficient number of BSs. Conventional minimum values on the number of BSs required for the presence of an unambiguous solution to the localization problem in the $\mathbb{R}^{2}$ plane are 2, 3, and 4 for triangulation (e.g. AOA), trilateration (e.g., TOA and RSS), and multilateration (e.g., TDOA) techniques, respectively.

For the general collaborative positioning problem, the conditions required for a device to be located are much more complicated and require a topological analysis of the network as a whole [24]. For the problem setup in this paper, that of one MD collaborating with a second $\mathrm{MD}$, the conditions required for a given device to be uniquely localizable are presented in the following proposition.

Proposition 1 (Two-device collaborative localizability). A device $\mathbf{u}$ capable of collaborating with a second device $\mathbf{v}$ is uniquely localizable in $\mathbb{R}^{2}$ iff one of the following conditions is met:

$\mathbf{L 1} \mathbf{u}$ is directly connected to at least three BSs

$\mathbf{L 2} \mathbf{u}$ is directly connected to two BSs, $\mathbf{v}$ is directly connected to at least two BSs, and combined, $\mathbf{u}$ and $\mathbf{v}$ are connected to at least three unique noncollinear BSs. 
Proof. See [25].

As mentioned in [23], the above conditions provide (i) a generic characterization of unique localizability and (ii) assume error-free ranging observations. Regarding (i), the conditions hold for almost all configurations of network devices placed using our PPP models, since the randomization causes degenerate configurations to appear with zero probability in a continuous space. Regarding (ii), we note that this is essentially required in order to derive the localizability conditions. While errors may introduce degenerate cases, we will assume that they do not introduce additional global minima into the cost function. Lastly, note that Condition L1 above, which does not involve the secondary device $\mathbf{v}$, is the only condition for (and is thus the definition of) noncollaborative unique localizability.

\section{UNIQUE LOCALIZABILITY IMPROVEMENT}

Using the conditions presented in Proposition 1, we now proceed with our analysis of the impact a single collaborative link has on a device's ability to locate itself without ambiguity. Let $\mathbb{N}_{\mathbf{z}}=\sum_{x \in \Phi} \mathbb{1}\left(\operatorname{SINR}_{x \rightarrow \mathbf{z}} \geq \beta\right)$ represent the number of BSs hearable at some device $\mathrm{z}$. If $\mathcal{L}_{\mathrm{nc}}$ represents the event that device $\mathbf{u}$ is capable of localizing itself using only its BS connections, then $\mathbb{P}\left(\mathcal{L}_{\mathrm{nc}}\right)=\mathbb{P}\left(\mathbb{N}_{\mathbf{u}} \geq \ell+1\right)$, where $\ell=2$ in the case of ranging observations to BSs (Condition L1) ${ }^{1}$. Now, let $\mathcal{S}_{\mathbf{z}}^{[\ell]}$ represent the set of $\ell$ BSs whose signals arrive with the highest SINRs at some device $\mathbf{z}$. Then, the probability of $\mathcal{L}_{\mathrm{c}}$, the event that device $\mathbf{u}$ is localizable when collaborating with a second device $\mathbf{v}$, is

$\mathbb{P}\left(\mathcal{L}_{\mathrm{c}}\right)$

$=\mathbb{P}\left(\mathcal{L}_{\mathrm{nc}}\right)+\sum_{n=2}^{\infty} \mathbb{P}\left(\mathbb{N}_{\mathbf{u}}=2, \mathbb{N}_{\mathbf{v}}=n,\left\|\mathcal{S}_{\mathbf{u}}^{[2]} \cup \mathcal{S}_{\mathbf{v}}^{[n]}\right\| \geq 3\right)$

$\stackrel{(a)}{=} \mathbb{P}\left(\mathcal{L}_{\mathrm{nc}}\right)+\ldots$

$\sum_{n=2}^{\infty} \mathbb{P}\left(\mathbb{N}_{\mathbf{u}}=2, \mathbb{N}_{\mathbf{v}}=n,\left\|\mathcal{S}_{\mathbf{u}}^{[2]} \cup \mathcal{S}_{\mathbf{v}}^{[n]}\right\| \geq 3 \mid \mathcal{S}_{\mathbf{u}}^{[2]} \neq \mathcal{S}_{\mathbf{v}}^{[2]}\right) \ldots$

$\times \mathbb{P}\left(\mathcal{S}_{\mathbf{u}}^{[2]} \neq \mathcal{S}_{\mathbf{v}}^{[2]}\right)+\ldots$

$\sum_{n=2}^{\infty} \mathbb{P}\left(\mathbb{N}_{\mathbf{u}}=2, \mathbb{N}_{\mathbf{v}}=n,\left\|\mathcal{S}_{\mathbf{u}}^{[2]} \cup \mathcal{S}_{\mathbf{v}}^{[n]}\right\| \geq 3 \mid \mathcal{S}_{\mathbf{u}}^{[2]}=\mathcal{S}_{\mathbf{v}}^{[2]}\right) \ldots$

$\times \mathbb{P}\left(\mathcal{S}_{\mathbf{u}}^{[2]}=\mathcal{S}_{\mathbf{v}}^{[2]}\right)$

$\stackrel{(b)}{=} \mathbb{P}\left(\mathcal{L}_{\mathrm{nc}}\right)+\sum_{n=2}^{\infty} \mathbb{P}\left(\mathbb{N}_{\mathbf{u}}=2, \mathbb{N}_{\mathbf{v}}=n \mid \mathcal{S}_{\mathbf{u}}^{[2]} \neq \mathcal{S}_{\mathbf{v}}^{[2]}\right) \mathbb{P}\left(\mathcal{S}_{\mathbf{u}}^{[2]} \neq \mathcal{S}_{\mathbf{v}}^{[2]}\right.$

$+\sum_{k=1}^{\infty} \mathbb{P}\left(\mathbb{N}_{\mathbf{u}}=2, \mathbb{N}_{\mathbf{v}}=2+k \mid \mathcal{S}_{\mathbf{u}}^{[2]}=\mathcal{S}_{\mathbf{v}}^{[2]}\right) \mathbb{P}\left(\mathcal{S}_{\mathbf{u}}^{[2]}=\mathcal{S}_{\mathbf{v}}^{[2]}\right)$

where $(a)$ follows from the law of total probability and Bayes' rule, and (b) follows from (i) the fact that $\mathbb{N}_{\mathbf{u}}=2, \mathbb{N}_{\mathbf{v}}=n \geq$ 2, and $\mathcal{S}_{\mathbf{u}}^{[2]} \neq \mathcal{S}_{\mathbf{v}}^{[2]}$ imply that $\left\|\mathcal{S}_{\mathbf{u}}^{[2]} \cup \mathcal{S}_{\mathbf{v}}^{[n]}\right\| \geq 3$, (ii) the fact

\footnotetext{
${ }^{1}$ Note that the work in this paper can be extended to consider range-difference observations from BSs by setting $\ell=3$ as in [25].
}

that $\mathcal{S}_{\mathbf{u}}^{[2]}=\mathcal{S}_{\mathbf{v}}^{[2]}$ implies that $\left\|\mathcal{S}_{\mathbf{u}}^{[2]} \cup \mathcal{S}_{\mathbf{v}}^{[2]}\right\| \nsucceq 3$, and (iii) from a simple rewriting of the lower limit in the second summation.

\section{A. The no shadowing case}

First, we consider the scenario without shadowing. When shadowing is absent, i.e., $\mathcal{F}_{g \rightarrow h}=1$ in (1) for all $g$ and $h$, ranking the BSs by decreasing SINRs is equivalent to ranking them by increasing distances from the receiver. Thus, there is a strong correlation between the hearable BSs at two nearby devices and

$$
\mathbb{P}\left(\mathbb{N}_{\mathbf{u}}=2, \mathbb{N}_{\mathbf{v}}=2+k \mid \mathcal{S}_{\mathbf{u}}^{[2]}=\mathcal{S}_{\mathbf{v}}^{[2]}\right) \rightarrow 0
$$

quickly as $k$ increases. Intuitively, a hearability mismatch of $k$ BSs is unlikely, even for small values of $k$, when the closest two BSs to $\mathbf{u}$ and $\mathbf{v}$ are conditioned to be the same. We now remove this term by letting $\mathbb{P}\left(\mathbb{N}_{\mathbf{u}}=2, \mathbb{N}_{\mathbf{v}}=2+k \mid \mathcal{S}_{\mathbf{u}}^{[2]}=\mathcal{S}_{\mathbf{v}}^{[2]}\right)=0$ since $k \geq 1$ and approximate (3) as

$$
\begin{aligned}
\mathbb{P}\left(\mathcal{L}_{\mathrm{c}}\right) \approx \mathbb{P}\left(\mathcal{L}_{\mathrm{nc}}\right) & +\sum_{n=2}^{\infty} \mathbb{P}\left(\mathbb{N}_{\mathbf{u}}=2, \mathbb{N}_{\mathbf{v}}=n \mid \mathcal{S}_{\mathbf{u}}^{[2]} \neq \mathcal{S}_{\mathbf{v}}^{[2]}\right) \\
& \times \mathbb{P}\left(\mathcal{S}_{\mathbf{u}}^{[2]} \neq \mathcal{S}_{\mathbf{v}}^{[2]}\right) .
\end{aligned}
$$

The following assumption will further simplify our analysis.

Assumption 1 (Independent base station hearability). When two devices have different sets of two strongest base stations, their joint hearability probability may be calculated as the product of their individual hearability probabilities:

$\mathbb{P}\left(\mathbb{N}_{\mathbf{u}}=m, \mathbb{N}_{\mathbf{v}}=n \mid \mathcal{S}_{\mathbf{u}}^{[2]} \neq \mathcal{S}_{\mathbf{v}}^{[2]}\right)=\mathbb{P}\left(\mathbb{N}_{\mathbf{u}}=m\right) \mathbb{P}\left(\mathbb{N}_{\mathbf{v}}=n\right)$.

Under Assumption 1, we arrive at the following final expression for $\mathbb{P}\left(\mathcal{L}_{\mathrm{c}}\right)$ in (4):

$$
\begin{aligned}
& \mathbb{P}\left(\mathcal{L}_{\mathrm{c}}\right) \approx \mathbb{P}\left(\mathcal{L}_{\mathrm{nc}}\right)+\sum_{n=2}^{\infty} \mathbb{P}\left(\mathbb{N}_{\mathbf{u}}=2\right) \mathbb{P}\left(\mathbb{N}_{\mathbf{v}}=n\right) \mathbb{P}\left(\mathcal{S}_{\mathbf{u}}^{[2]} \neq \mathcal{S}_{\mathbf{v}}^{[2]}\right) \\
& =\mathbb{P}\left(\mathbb{N}_{\mathbf{u}} \geq 3\right)+\mathbb{P}\left(\mathbb{N}_{\mathbf{u}}=2\right) \mathbb{P}\left(\mathbb{N}_{\mathbf{v}} \geq 2\right) \mathbb{P}\left(\mathcal{S}_{\mathbf{u}}^{[2]} \neq \mathcal{S}_{\mathbf{v}}^{[2]}\right) .
\end{aligned}
$$

Exact expressions for the hearability terms in (5) (i.e., all terms except $\left.\mathbb{P}\left(\mathcal{S}_{\mathbf{u}}^{[2]} \neq \mathcal{S}_{\mathbf{v}}^{[2]}\right)\right)$ are presented in [26]. The exact calculations, however, are extremely involved and time consuming, leading us to employ the approximations presented in [20], which are nearly indistinguishable from truth. Specifically, $\mathbb{P}\left(\mathbb{N}_{\mathbf{z}} \geq L\right)=\mathrm{P}_{\mathrm{L}}(1,1, \alpha, \beta, 1, \lambda)$, where the right-hand term is presented in Theorem 2 of [20]. In the following section, we will derive an exact expression for the remaining term.

\section{B. Probability that two devices share the same set of closest $B S s$}

Recall that MD $\mathbf{u}$ is the device whose localizability is being directly considered and MD $\mathbf{v}$ is a secondary device with whom $\mathbf{u}$ is able to collaborate. Without loss of generality, let $\mathbf{u}=\left[\begin{array}{ll}0 & 0\end{array}\right]^{\top}$ be located at the origin and $\mathbf{v}$ be a random distance $D$ away and located at $\mathbf{v}=\left[\begin{array}{ll}D & 0\end{array}\right]^{\top}$. We begin our derivation of $\mathbb{P}\left(\mathcal{S}_{\mathbf{u}}^{[2]} \neq \mathcal{S}_{\mathbf{v}}^{[2]}\right)$ by first conditioning on $D=d$ and presenting the following shape. 


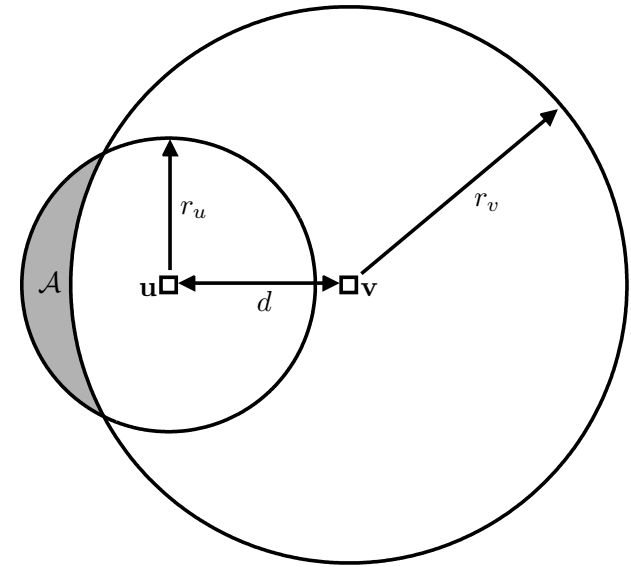

Fig. 1. A LUNE is formed by the region of one circle which is outside its intersection with another partially-overlapping circle.

Definition 2 (Lune). Consider two partially-overlapping circles with radii $r_{u}$ and $r_{v}$ whose centers are separated by distance $d$, as shown in Figure 1. Region $\mathcal{A}$ is called a lune and its area is [27]

$$
\begin{aligned}
& \mathbb{A}_{\mathbb{B}}\left(r_{u}, r_{v}, d\right)=\frac{1}{2} \sqrt{\mathcal{A}_{1} \cdot \mathcal{A}_{2} \cdot \mathcal{A}_{3} \cdot \mathcal{A}_{4}} \\
& +r_{u}^{2} \sec ^{-1}\left(\frac{2 d r_{u}}{r_{v}^{2}-r_{u}^{2}-d^{2}}\right)-r_{v}^{2} \sec ^{-1}\left(\frac{2 d r_{v}}{r_{v}^{2}+d^{2}-r_{u}^{2}}\right),
\end{aligned}
$$

where $\mathcal{A}_{1}=r_{u}+r_{v}+d, \mathcal{A}_{2}=r_{v}+d-r_{u}, \mathcal{A}_{3}=d+r_{u}-r_{v}$, and $\mathcal{A}_{4}=r_{u}+r_{v}-d$.

In addition to $\mathbb{A}_{\mathbb{B}}(\cdot)$, let $\mathbb{A}_{\odot}(r)=\pi r^{2}$ return the well-known area of a circle with radius $r$.

Lemma 1. The probability that $\mathbf{u}$ and $\mathbf{v}$, separated by distance $d$, have the same set of $\ell$ closest base stations, while also having the same $\ell^{\text {th }}$ closest $B S$, is

$$
\begin{aligned}
& \mathbb{P}\left(\mathcal{S}_{\mathbf{u}}^{[\ell]}=\mathcal{S}_{\mathbf{v}}^{[\ell]}, \mathcal{S}_{\mathbf{u}}^{[\ell-1]}=\mathcal{S}_{\mathbf{v}}^{[\ell-1]} \mid D=d\right)=\frac{2}{\pi(\ell-1) !} \\
& \times \int_{0}^{\infty} \int_{0}^{\pi}\left(\frac{\mathbb{A}_{\bigcirc}(r)-\mathbb{A}_{\mathbb{Q}}\left(r, \sqrt{r^{2}+d^{2}-2 r d \cos \theta}, d\right)}{\mathbb{A}_{\circ}(r)}\right)^{\ell-1} \\
& \times e^{-\lambda\left(\mathbb{A}_{\mathbb{Q}}\left(\sqrt{r^{2}+d^{2}-2 r d \cos \theta}, r, d\right)+\pi r^{2}\right)} \frac{\left(\lambda \pi r^{2}\right)^{\ell}}{r} \mathrm{~d} \theta \mathrm{d} r .
\end{aligned}
$$

Proof. See [25].

Lemma 2. The probability that $\mathbf{u}$ and $\mathbf{v}$, separated by distance $d$, have the same set of $\ell$ closest BSs, while differing in their $\ell^{\text {th }}$ closest BSs, is

$$
\begin{aligned}
& \mathbb{P}\left(\mathcal{S}_{\mathbf{u}}^{[\ell]}=\mathcal{S}_{\mathbf{v}}^{[\ell]}, \mathcal{S}_{\mathbf{u}}^{[\ell-1]} \neq \mathcal{S}_{\mathbf{v}}^{[\ell-1]} \mid D=d\right)=\frac{2(\ell-1)}{\pi(\ell-1) !} \\
& \times \int_{0}^{\infty} \frac{1}{\mathbb{A}_{\odot}(r)} \int_{0}^{\pi} \int_{\sqrt{r^{2}+d^{2}-2 r d \cos \theta}}^{d+r}\left(\frac{\mathbb{A}_{\odot}(r)-\mathbb{A}_{\mathbb{}}(r, x, d)}{\mathbb{A}_{\odot}(r)}\right)^{\ell-2} \\
& \times e^{-\lambda\left(\mathbb{A}_{\mathbb{}}(x, r, d)+\pi r^{2}\right)} \phi_{\text {range }}(d, r, x) \frac{x\left(\lambda \pi r^{2}\right)^{\ell}}{r} \mathrm{~d} x \mathrm{~d} \theta \mathrm{d} r,
\end{aligned}
$$

where

$$
\phi_{\text {range }}(d, r, x)=2 \cos ^{-1}\left(\frac{d^{2}+x^{2}-r^{2}}{2 \cdot d \cdot x}\right) .
$$

Proof. See [25].

Combining the two lemmas, we arrive at the following theorem.

Theorem 1. The probability that two devices $\mathbf{u}$ and $\mathbf{v}$, separated by distance $d$, share the same set of $\ell$ closest BSs is

$$
\begin{gathered}
\mathbb{P}\left(\mathcal{S}_{\mathbf{u}}^{[\ell]}=\mathcal{S}_{\mathbf{v}}^{[\ell]} \mid D=d\right)=\mathbb{P}\left(\mathcal{S}_{\mathbf{u}}^{[\ell]}=\mathcal{S}_{\mathbf{v}}^{[\ell]}, \mathcal{S}_{\mathbf{u}}^{[\ell-1]}=\mathcal{S}_{\mathbf{v}}^{[\ell-1]} \mid D=d\right) \\
+\mathbb{P}\left(\mathcal{S}_{\mathbf{u}}^{[\ell]}=\mathcal{S}_{\mathbf{v}}^{[\ell]}, \mathcal{S}_{\mathbf{u}}^{[\ell-1]} \neq \mathcal{S}_{\mathbf{v}}^{[\ell-1]} \mid D=d\right) .
\end{gathered}
$$

Proof. By the law of total probability, the probability that the two mobile devices have the same set of $\ell$ closest BSs is simply the sum of the probabilities presented in Lemmas 1 and 2 .

Corollary 1.1. When devices $\mathbf{u}$ and $\mathbf{v}$ (separated by distance d) both successfully hear exactly $\ell$ base stations, the probability that collaboration between them will result in a combined hearability of at least $\ell+1$ unique base stations is

$$
\begin{gathered}
\mathbb{P}\left(\left\|\mathcal{S}_{\mathbf{u}}^{[\ell]} \cup \mathcal{S}_{\mathbf{v}}^{[\ell]}\right\| \geq \ell+1 \mid D=d\right)=\mathbb{P}\left(\mathcal{S}_{\mathbf{u}}^{[\ell]} \neq \mathcal{S}_{\mathbf{v}}^{[\ell]} \mid D=d\right) \\
=1-\mathbb{P}\left(\mathcal{S}_{\mathbf{u}}^{[\ell]}=\mathcal{S}_{\mathbf{v}}^{[\ell]} \mid D=d\right) .
\end{gathered}
$$

In order to endow $D$ with a distribution, let us now consider device $\mathbf{v}$ to be the $K^{\text {th }}$ closest MD to $\mathbf{u}$. Since the MDs are modeled according to a homogeneous PPP with density $\nu$, it follows from Slivnyak's theorem [13] that the distribution of the distance from any device to its $K^{\text {th }}$ neighbor, $D=D_{K}$, is [28]

$$
f_{D_{K}}(d ; K, \nu)=e^{-\nu \pi d^{2}} \frac{2\left(\nu \pi d^{2}\right)^{K}}{d(K-1) !} .
$$

Clearly, $K=1$ represents a case of particular interest, i.e., $\mathbf{u}$ collaborates with its closest neighbor. Now, for the general $K^{\text {th }}$ neighbor setup, we arrive at the following theorem.

Theorem 2. The probability that device $\mathbf{u}$ and its $K^{\text {th }}$ closest neighboring device $\mathbf{v}$ share the same set of $\ell$ closest base stations is

$$
\begin{aligned}
\mathbb{P}\left(\mathcal{S}_{\mathbf{u}}^{[\ell]}=\mathcal{S}_{\mathbf{v}}^{[\ell]}\right)= & \frac{2}{(K-1) !} \int_{0}^{\infty} \mathbb{P}\left(\mathcal{S}_{\mathbf{u}}^{[\ell]}=\mathcal{S}_{\mathbf{v}}^{[\ell]} \mid D=y\right) \\
& \times e^{-\nu \pi y^{2}} \frac{\left(\nu \pi y^{2}\right)^{K}}{y} \mathrm{~d} y
\end{aligned}
$$

Proof. The result is obtained by deconditioning (9) on $D=$ $D_{K}$, i.e., $\mathbb{P}\left(\mathcal{S}_{\mathbf{u}}^{[\ell]}=\mathcal{S}_{\mathbf{v}}^{[\ell]}\right)=\mathbb{E}_{D_{K}}\left[\mathbb{P}\left(\mathcal{S}_{\mathbf{u}}^{[\ell]}=\mathcal{S}_{\mathbf{v}}^{[\ell]} \mid D_{K}\right)\right]$.

Corollary 2.1. Conditioned on device $\mathbf{u}$ and its $K^{\text {th }}$ closest neighbor $\mathbf{v}$ both successfully hearing exactly $\ell$ base stations, the probability that collaboration among them will lead to a combined hearability of at least $\ell+1$ unique base stations is

$$
\begin{aligned}
\mathbb{P}\left(\left\|\mathcal{S}_{\mathbf{u}}^{[\ell]} \cup \mathcal{S}_{\mathbf{v}}^{[\ell]}\right\| \geq \ell+1\right) & =\mathbb{P}\left(\mathcal{S}_{\mathbf{u}}^{[\ell]} \neq \mathcal{S}_{\mathbf{v}}^{[\ell]}\right) \\
& =1-\mathbb{P}\left(\mathcal{S}_{\mathbf{u}}^{[\ell]}=\mathcal{S}_{\mathbf{v}}^{[\ell]}\right) .
\end{aligned}
$$

Finally, (13) in Corollary 2.1 provides the exact expression for $\mathbb{P}\left(\mathcal{S}_{\mathbf{u}}^{[2]} \neq \mathcal{S}_{\mathbf{v}}^{[2]}\right)$ in (5), which, when combined with the 
hearability results in [20], yields $\mathbb{P}\left(\mathcal{L}_{\mathrm{c}}\right)$, the probability of unique localizability in the collaborative scenario.

\section{The shadowing case}

Now, we consider the unique localizability problem in the presence of log-normal shadowing. The difficulty in analyzing this scenario lies in the fact that, unlike in the no shadowing case, the set of two strongest BSs at some device $\mathbf{z}, \mathcal{S}_{\mathbf{z}}^{[2]}$, is no longer directly tied to the two geographically closest BSs to $\mathbf{z}$. Thus, we cannot use the geometric analysis of the previous section for comparing the sets of strongest BSs at two MDs. Instead, we note that $\mathbb{P}\left(\left\|\mathcal{S}_{\mathbf{u}}^{[2]} \cup \mathcal{S}_{\mathbf{v}}^{[2]}\right\| \geq 3 \mid D=d\right)=$ $\mathbb{P}\left(\mathcal{S}_{\mathbf{u}}^{[2]} \neq \mathcal{S}_{\mathbf{v}}^{[2]} \mid D=d\right) \rightarrow 1$ for all $d$ as the shadowing standard deviation $\sigma_{s}$ increases. In order to get an initial tractable expression for the shadowing case, we then use the simplifying assumption that $\mathbb{P}\left(\mathcal{S}_{\mathbf{u}}^{[2]} \neq \mathcal{S}_{\mathbf{v}}^{[2]}\right)=1$ and invoke Assumption 1 to arrive at the following approximation of (3) for the shadowing case:

$$
\begin{aligned}
\mathbb{P}\left(\mathcal{L}_{\mathrm{c}}\right) & \approx \mathbb{P}\left(\mathcal{L}_{\mathrm{nc}}\right)+\sum_{n=2}^{\infty} \mathbb{P}\left(\mathbb{N}_{\mathbf{u}}=2\right) \mathbb{P}\left(\mathbb{N}_{\mathbf{v}}=n\right) \\
& =\mathbb{P}\left(\mathbb{N}_{\mathbf{u}} \geq 3\right)+\mathbb{P}\left(\mathbb{N}_{\mathbf{u}}=2\right) \mathbb{P}\left(\mathbb{N}_{\mathbf{v}} \geq 2\right) .
\end{aligned}
$$

A more exact expression for (3) in the shadowing case is outside the scope of this paper. Nevertheless, it will be evident in the following section that (14) is surprisingly accurate.

\section{Numerical Results AND Discussion}

In this section, we present numerical results and use them to draw insights into the value of collaboration for improving unique localizability. We begin by focusing on the no shadowing case and taking a look at the number of unique BSs among the closest BSs at two devices.

\section{A. Sufficient unique base stations versus collaborator separa- tion}

Let $\mathbf{u}$ and $\mathbf{v}$ be two devices separated by distance $d$ as described in Section IV-B. When both devices successfully hear exactly two BSs, neither is localizable per the conditions presented in Proposition 1. The key differentiator in determining whether collaboration between these devices will be beneficial to localizability is whether or not the two devices hear a combined three or more unique BSs. When $d$ is fixed, it is the density of the BSs which will affect the probability of obtaining a sufficient number of unique BSs. This is illustrated in Fig. 2, where (10) in Corollary 1.1 is plotted versus $d$ for various BS densities $\lambda$. Note that the densities are multiples of the PPP density which results in the same average number of BSs per unit area as an infinite hexagonal grid with $500 \mathrm{~m}$ intersite distances (ISD). For a fixed separation $d$, it is obvious that a higher BS density leads to a greater likelihood that collaboration will be beneficial in this scenario. While collaboration with farther devices also increases this likelihood, Fig. 2 reveals that there is a certain distance beyond which it is not necessary to collaborate.

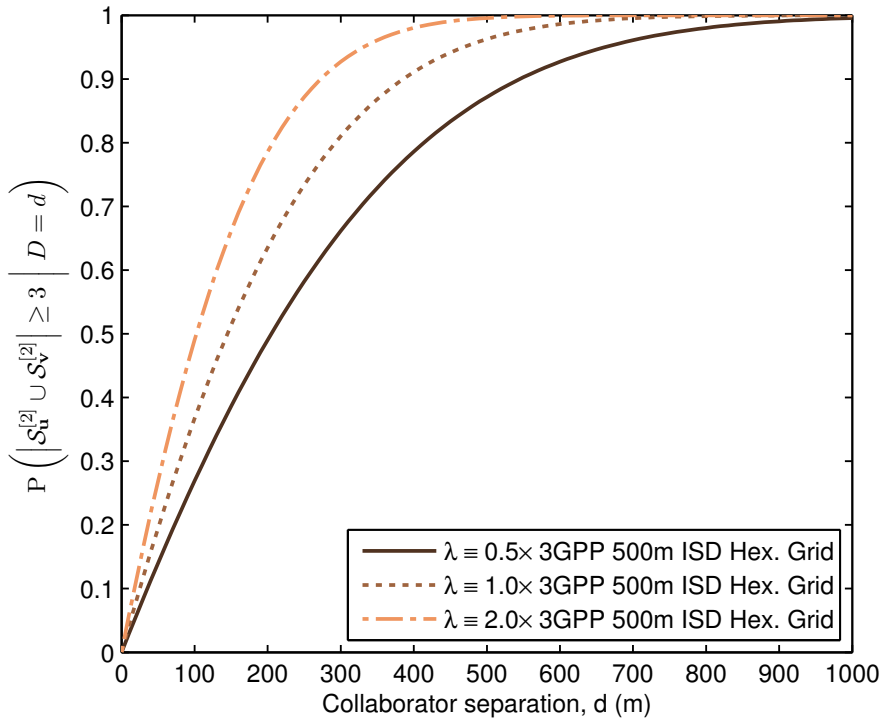

Fig. 2. Uniqueness Among Closest Nodes: The probability that two devices, separated by distance $d$ and each hearing exactly two BSs, will benefit in terms of their combined number of unique BSs (Corollary 1.1).

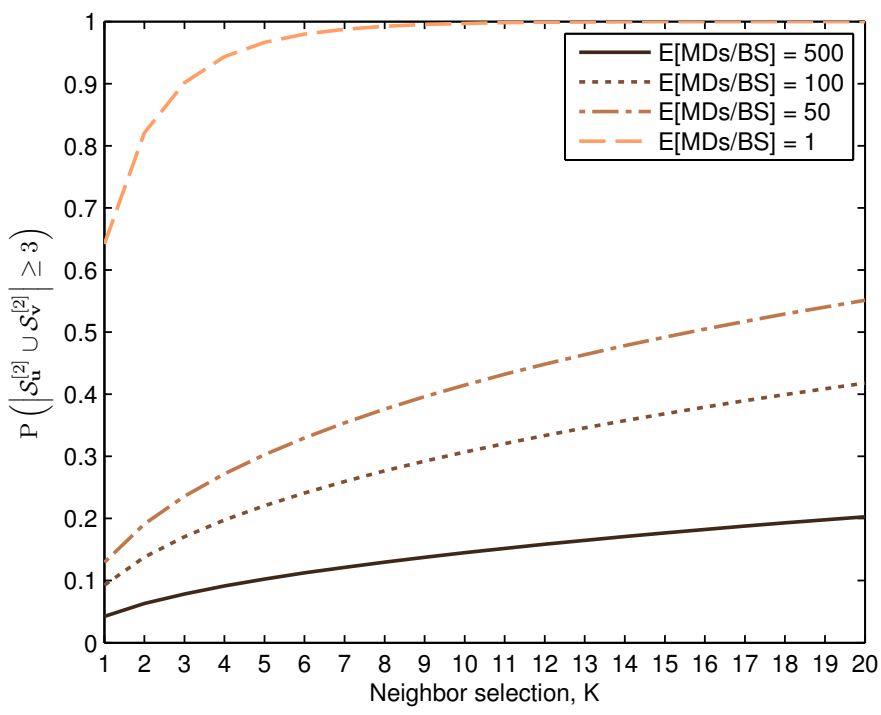

Fig. 3. The Impact of Neighbor Selection: The analysis of Corollary 2.1 reveals the benefit of selecting farther neighboring devices for increasing their combined number of unique BSs.

\section{B. Sufficient unique base stations versus collaborator selection}

Next, let $\mathbf{v}$ be the $K^{\text {th }}$ neighbor of $\mathbf{u}$ in the PPP of MDs $\Psi$. Given that both devices successfully hear exactly two BSs, Fig. 3 presents the probabilities that a collaborative link between $\mathbf{u}$ and $\mathbf{v}$ will be beneficial to their unique localizabilities for various MD densities (expressed as the average number of MDs per BS or cell). The results show that selecting the closest neighbor, with whom it may likely be easiest to collaborate, is not necessarily a good idea, especially when a MD is in the neighborhood of a large number of other MDs. The nearest neighbors are valuable when MD densities are low, but even then, selecting a farther neighbor is typically more beneficial 


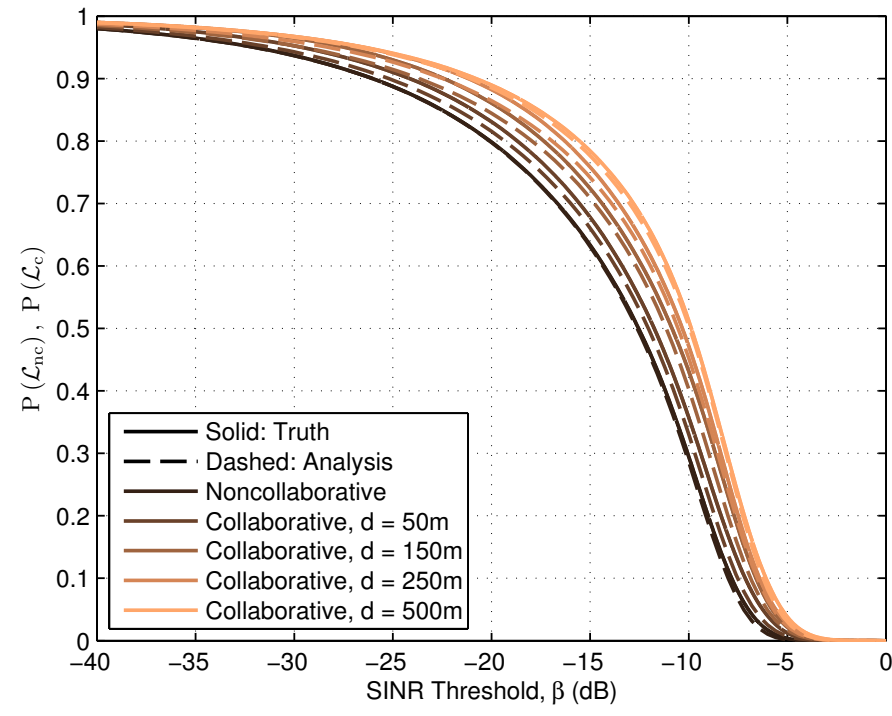

(a) Probability of unique localizability

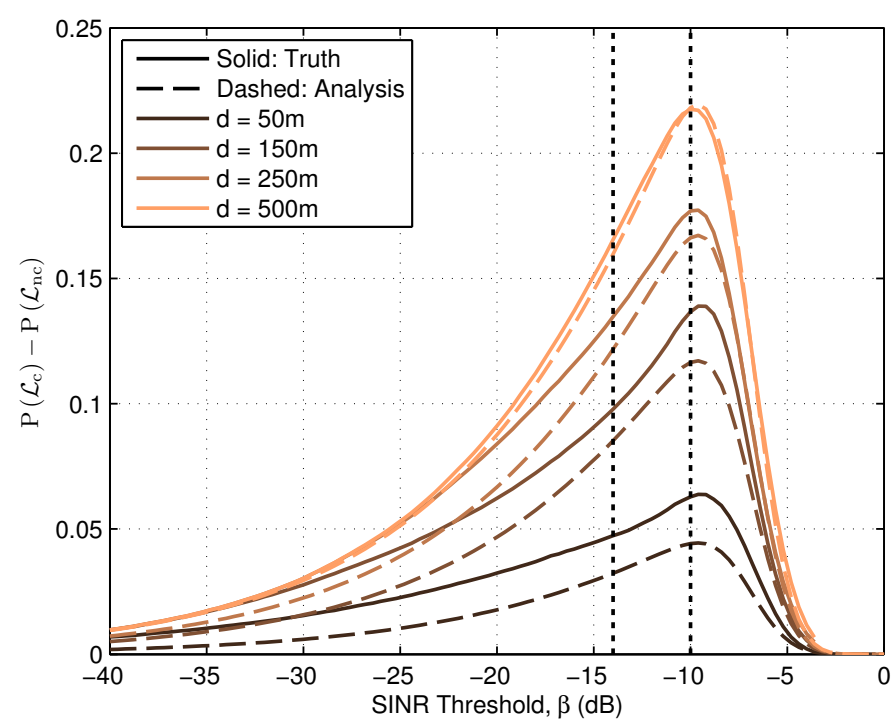

(b) Absolute increase in unique localizability probability

Fig. 4. UNIQUE LOCALIZABILITY in range-based positioning (e.g., using RSS or TOA observations) for various collaborator separations $d$. The separation plays a major role in how beneficial collaboration will be. Note that the dotted lines at $\beta=-10$ and $-14 \mathrm{~dB}$ delineate the range of SINR threshold values considered in 3GPP for far away BSs, which is just below where the peak collaborative benefits are obtained. $(\alpha=4$.)

(up to a point). By revisiting (11), it becomes clear that higher values of $K$ and lower values of $\nu$ lead to greater probabilities of longer distances separating $\mathbf{u}$ and $\mathbf{v}$. Thus, we see that ultimately, it is the separation between collaborators that is the driving force behind determining the value of collaboration, which leads us to present all subsequent results in light of the distance $d$ separating the collaborating devices.

\section{Probability of unique localizability}

Now, we consider the benefit of collaboration to localizability and how it is impacted by the SINR threshold $\beta$. For $\alpha=4$ (which we consider throughout as it is close to the 3.76 value used in 3GPP positioning studies [21] and allows the use of

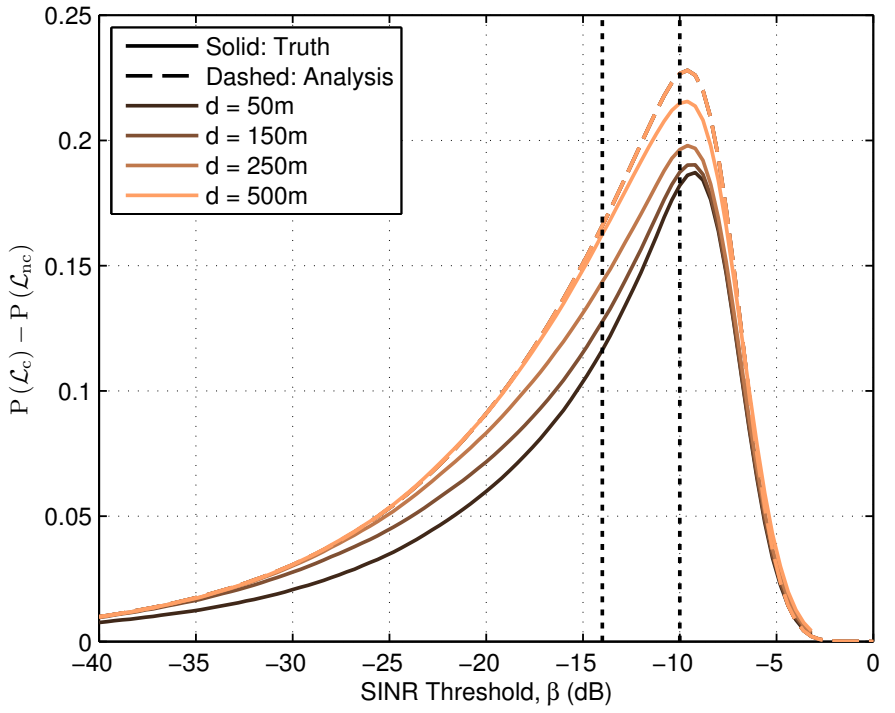

Fig. 5. THE IMPACT OF SHADOWING on the probability of unique localizability.

simplified expressions from [20]) and various collaborator separations $d$, Fig. 4 shows (a) the probability of unique localizability and (b) the absolute increase in these probabilities provided through device $\mathbf{u}$ 's collaborative link with device $\mathbf{v}$. We note that the approximation in (5) is within an absolute error of 0.02 from the truth, which is gathered via simulation. Furthermore, we see that the benefit from collaboration is a non-monotonic function of $\beta$. Moreover, $\beta=-9 \mathrm{~dB}$ appears to be a sweet spot which maximizes the collaborative benefit, providing an approximately $6 \%$ to $22 \%$ absolute increase in the probabilities of unique localizability. Randomization due to the presence of shadowing, which is a more applicable scenario for cellular positioning, may actually help matters, as discussed next.

\section{The impact of shadowing on localizability}

Next, we consider the impact of shadowing using log-normal shadowing with a standard deviation of $\sigma_{s}=8 \mathrm{~dB}$ and a correlation of 0.5 between the signals received at two devices originating from the same BS. Recall that (14) was derived using some very simplifying assumptions, including that two devices each hearing two BSs certainly hear at least three unique BSs, independent of the separation distance. Fig. 5 reveals that this was, in fact, not a bad assumption. The separation between the devices plays a highly-reduced role in the localizability probability compared to its role in the no shadowing case. Moreover, it is observed that the presence of shadowing is quite beneficial, primarily for increasing the probability that collaboration will improve localizability for shorter device separations $d$. For all values of $d$, the benefit from collaboration is relatively similar, which is quite different from the no shadowing case.

\section{CONCLUSION}

In this paper, we presented an analysis of the impact of a single collaborative link on the probability of a mobile 
device being able to locate itself without ambiguity (i.e., being uniquely localizable). This is in contrast to previous works, which have relied on simulations to study collaborative positioning in similar setups which include network selfinterference. In the absence of shadowing, the results show that collaboration is beneficial for increasing the probability of unique localizability in range-based positioning systems and the key element influencing the extent of the benefit is the separation between the devices. Shadowing reduces this distance-dependence, thereby greatly increasing the benefit obtained from close collaborators. These results are significant and demonstrate that short-distance small-scale collaboration, which is the most reasonable scenario for cellular networks, is a very worthwhile pursuit and likely to be a significant aid in the fight against the hearability problem.

\section{REFERENCES}

[1] Y. Ko and N. H. Vaidya, "Location-aided routing (LAR) in mobile ad-hoc networks," Wirel. Networks, vol. 6, no. 4, pp. 307-321, 2000.

[2] R. Jain, A. Puri, and R. Sengupta, "Geographical routing using partial information for wireless ad-hoc networks," IEEE Pers. Commun., vol. 8, no. 1, pp. 48-57, 2001.

[3] L. Blazevic, S. Giordano, and J.-Y. Le Boudec, "Self-organized routing in wide area mobile ad-hoc networks," in Proc. IEEE Glob. Telecommun. Conf., vol. 5, 2001, pp. 2814-2818.

[4] N. Patwari and J. N. Ash, "Locating the nodes: cooperative localization in wireless sensor networks," IEEE Signal Process. Mag., pp. 54-69, Jul. 2005.

[5] A. H. Sayed, A. Tarighat, and N. Khajehnouri, "Network-based wireless location: challenges faced in developing techniques for accurate wireless location information," IEEE Signal Process. Mag., vol. 22, no. 4, pp 24-40, Jul. 2005.

[6] Federal Communications Commission, "Wireless E911 location accuracy requirements," PS Docket No. 07-114, Jan. 2015.

[7] Third Generation Partnership Project (3GPP), "R1-091912: Discussions on UE positioning issues," Nortel, 3GPP TSG-RAN WG1 \#57, San Francisco, USA, May 2009.

[8] A. Savvides, C.-C. Han, and M. B. Strivastava, "Dynamic fine-grained localization in ad-hoc networks of sensors," in Proc. ACM Int. Conf. Mob. Comput. Netw., New York, New York, USA, Jul. 2001, pp. 166-179.

[9] S. Gezici, G. Giannakis, H. Kobayashi, A. Molisch, H. V. Poor, and Z. Sahinoglu, "Localization via ultra-wideband radios: a look at positioning aspects for future sensor networks," IEEE Signal Process. Mag., vol. 22, no. 4, pp. 70-84, Jul. 2005.

[10] R. M. Vaghefi and R. M. Buehrer, "Improving positioning in LTE through collaboration," in Proc. Work. Positioning, Navig. Commun., Mar. 2014.

[11] H. Wymeersch, J. Lien, and M. Z. Win, "Cooperative localization in wireless networks," Proc. IEEE, vol. 97, no. 2, pp. 427-450, Feb. 2009.

[12] J. Schloemann and R. M. Buehrer, "On the value of collaboration in location estimation," to appear in IEEE Trans. Veh. Tech.

[13] M. Haenggi, Stochastic Geometry for Wireless Networks. New York: Cambridge University Press, 2013.

[14] A. T. Ihler, J. W. Fischer III, R. L. Moses, and A. S. Willsky, "Nonparametric belief propagation for self-localization of sensor networks," IEEE J. Sel. Areas Commun., vol. 23, no. 4, pp. 809-819, 2005.

[15] H. Wymeersch, U. Ferner, and M. Z. Win, "Cooperative Bayesian selftracking for wireless networks," IEEE Commun. Lett., vol. 12, no. 7, pp. 505-507, Jul. 2008

[16] T. Eren, O. Goldenberg, W. Whiteley, Y. Yang, A. Morse, B. Anderson, and P. Belhumeur, "Rigidity, computation, and randomization in network localization," in Proc. IEEE Int. Conf. Comput. Commun., vol. 4, 2004, pp. 2673-2684.

[17] Code of Federal Regulations, "911 Service," 47 C.F.R. 20.18(h)(2)(ii), 2015.

[18] J. G. Andrews, F. Baccelli, and R. K. Ganti, "A tractable approach to coverage and rate in cellular networks," IEEE Trans. Commun., vol. 59, no. 11 , pp. $3122-3134$, Nov. 2011.
[19] H. S. Dhillon, R. K. Ganti, F. Baccelli, and J. G. Andrews, "Modeling and analysis of K-tier downlink heterogeneous cellular networks," IEEE J. Sel. Areas Commun., vol. 30, no. 3, pp. 550-560, Apr. 2012.

[20] J. Schloemann, H. S. Dhillon, and R. M. Buehrer, "Towards a tractable analysis of localization fundamentals in cellular networks," arXiv:1502.06899 [cs.IT].

[21] Third Generation Partnership Project (3GPP), "R1-091443: Evaluation parameters for positioning studies," Alcatel-Lucent, Ericsson, Motorola Nokia, NSN, Nortel, Qualcomm Europe, 3GPP TSG-RAN WG1 \#56bis, Seoul, Korea, Mar. 2009.

[22] F. Daneshgaran, M. Laddomada, and M. Mondin, "Connection between system parameters and localization probability in network of randomly distributed nodes," IEEE Trans. Wirel. Commun., vol. 6, no. 12, pp. 4383-4389, Dec. 2007.

[23] D. K. Goldenberg, A. Krishnamurthy, W. C. Maness, Y. R. Yang, A. Young, A. S. Morse, A. Savvides, and B. D. O. Anderson, "Network localization in partially localizable networks," in Proc. IEEE Int. Conf. Comput. Commun., vol. 1, 2005, pp. 313-326.

[24] Z. Yang and Y. Liu, "Understanding node localizability of wireless adhoc and sensor networks," IEEE Trans. Mob. Comput., vol. 11, no. 8, pp. 1249-1260, Aug. 2012.

[25] J. Schloemann, H. S. Dhillon, and R. M. Buehrer, "An Analysis of the Improvement in Unique Localizability Through Collaboration," arXiv [cs.IT].

[26] H. P. Keeler, B. Blaszczyszyn, and M. K. Karray, "SINR-based k-coverage probability in cellular networks with arbitrary shadowing," in IEEE Int. Symp. Inf. Theory, Jul. 2013, pp. 1167-1171.

[27] E. W. Weisstein, "Lune," From MathWorld-A Wolfram Web Resource. http://mathworld.wolfram.com/Lune.html.

[28] M. Haenggi, "On distances in uniformly random networks," IEEE Trans. Inf. Theory, vol. 51, no. 10, pp. 3584-3586, Oct. 2005. 